\title{
O legado dos estudos críticos modernos em Jeremias
}

\section{The legacy of modern critical studies in Jeremiah}

\section{El legado de los estudios críticos modernos en Jeremías}

\author{
Anderson Yan*
}

\begin{abstract}
RESUMO
Esse ensaio argumenta que o estudo crítico em Jeremias não é algo que teve origem na modernidade apesar de que ela impulsionou essa empreitada trazendo consciência para a complexidade do longo processo composicional desse livro profético. Mesmo que grande parte dos seus pressupostos necessitam constante revisão, é inegável que o seu legado tenha proporcionado uma variedade de ferramentas e métodos robustos para a análise desse livro que permanece até os dias de hoje.

Palavras-chave: Jeremias; crítica textual; fonte; forma e redação; Deuteronomismo.
\end{abstract}

\begin{abstract}
This paper argues that criticism on Jeremiah did not have its origins in modernity, although it indeed triggered this enterprise raising awareness of the long complex process of composition of this prophetic book. Despite that significant part of the modern presupposition requires frequent review, one cannot doubt that its legacy provided a variation of thorough tools and methods for the analysis of this book, which stand still nowadays.

Keywords: Jeremiah; textual criticism; source; form and redaction; Deuteronomism.

\section{RESUMEN}

Este artículo sostiene que la crítica a Jeremías no tuvo sus orígenes en la modernidad, aunque de hecho provocó que esta empresa despertara la conciencia del largo y complejo proceso de composición de este libro profético. A pesar de que una parte importante de la presuposición moderna requiere una revisión frecuente, no se puede dudar de que su legado proporcionó una variedad de herramientas y métodos exhaustivos para el análisis de este libro, que aún se mantienen en pie en la actualidad.

Palabras clave: Jeremías; critica textual; fuente; forma y redacción; Deuteronomismo.
\end{abstract}

\section{Introdução}

Questões críticas sempre estiveram presentes nos estudos bíblicos demonstrando que a crítica bíblica não é algo exclusivo da modernidade, pois exegetas pré-modernos (p. ex., Calvino, Rashi, Kimhi, etc.) já demonstravam uma leitura relativamente sofisticada para o seu tempo ${ }^{1}$. Mais especificamente em relação ao livro atribuído ao profeta Jeremias, ${ }^{2}$ os Pais da Igreja já de-

\footnotetext{
* Doutorando em Bíblia Hebraica/Antigo Testamento pelo King’s College London.

E-mail: anderson.yan@gmail.com

1 Nesse sentido recomendo a leitura de ROGERSON, 1998, p. 6-24.

2 Daqui em diante Jeremias.
} 
monstravam ciência dos problemas textuais acerca das sagradas escrituras. Orígenes (184-253 EC) particularmente, apresenta um conhecimento detalhado das diferenças entre $\mathbb{G}$ e $\mathfrak{A t l}$-Jeremias ao relatar:

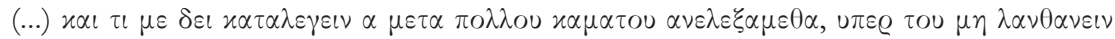

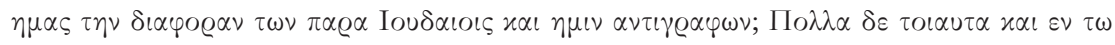

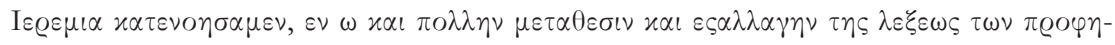

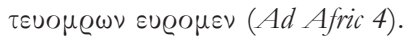

Posteriormente Jerônimo (347-419/20 EC) considerou essas diferenças como falha na história da transmissão do texto atribuindo ao erro dos copistas ao afirmar o seguinte:

(...) sed magis Jeremiae ordinem, librarierum errore confusum, multaque quae desuni, ex Hebraeis fontibus digerere, ne complere: ut novum ex veteri, verunique pro corrupto atque lalsato Prophetam teneas (Prolugus, PL 24, col. 679).

A relação entre Jeremias e a literatura Deuteronomista também não é algo que não passou despercebido pelos rabinos. Nesse ponto o Talmude (c. 500) preserva a organização pré-massorética dos livros da Bíblia מיאיבנ לש ורדס ןנבר ונת Hebraica (BH) colocando-os na seguinte ordem com a seguinte

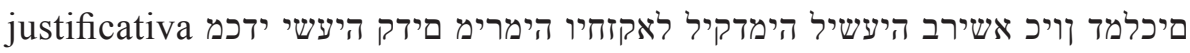

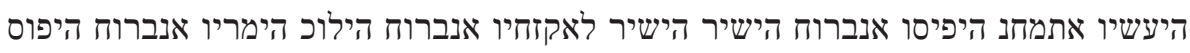

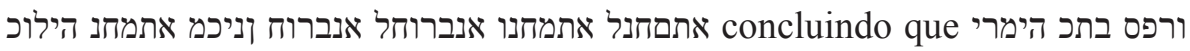

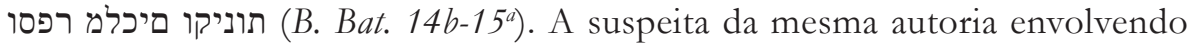
Reis e Jeremias provavelmente ocorre devido as semelhanças significativas apresentadas abaixo ${ }^{3}$ :

\section{Rs 25:27-30}

-דלמ ןיכיוהי תולגל הנש עבשו םישלשב יהיו

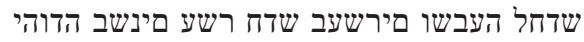

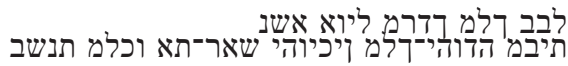
אסלכ לעמ ואסכ־תא ותיו תובט ותא רבדיו

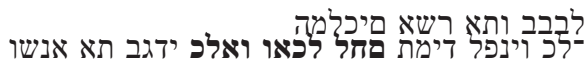

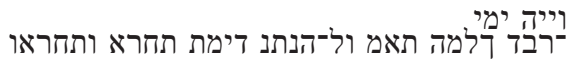
ויח ימי לכ ומויב םוי וליהנה
Jr 52:31-34 וכיוהי תולגל הנש עבשו מישלשב יהיו

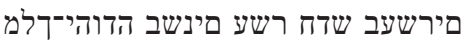

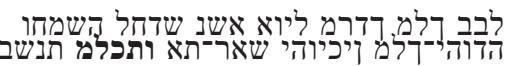
אילכה תיבמי ותוא אציוה שיות לעממ ואסכ־תא ןתיוו תובט ותא רבדיו

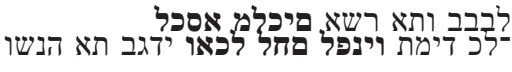

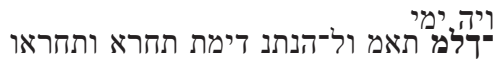
ימי לכ ותומ שויידע ומויב םוי־רבד לבב ותחו וימת

ויידיה

\footnotetext{
3 Diferenças entre as 2 Reis e Jeremias estão destacadas em negrito, porém elas praticamente desaparecem quando as comparações se baseiam na Vorlage de $\mathbb{6}$-J Jeremias.
} 
Não obstante, os exemplos citados acima não devem perturbar o leitor mais reverente as Sagradas Escrituras, pois Jeremias mesmo parece apresentar uma certa sustentação em relação ao papel ativo dos escribas no

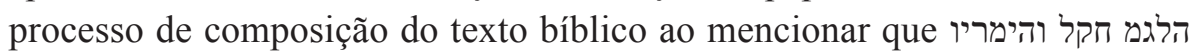

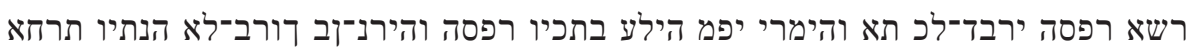

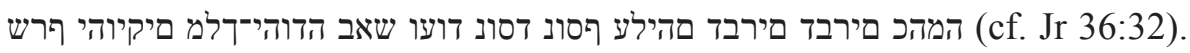

Assim, é razoável dizer que grande parte das questões críticas em relação ao texto não são fruto de uma pré-disposição para trazer descrédito dos críticos modernos, mas ao contrário, são questões que emergem da própria Bíblia 4 .

Esse ensaio procura apresentar um panorama das estruturas interpretativas aplicadas a Jeremias bem como as mudanças paradigmáticas aplicadas a esse livro profético. O enfoque será dado nos métodos diacrônicos excluindo abordagens sincrônicas mais recentes cuja agendas estão mais voltadas as preocupações no âmbito político, p. ex., etnias, gênero, ecologia, etc. (DAVIES, 2013, p. 1-10).

Apesar do termo "histórico-crítico" ser contestado devido ao fato de não refletir de forma integral a tarefa dos estudos bíblicos ao ponto de ser até mesmo questionado se pode ser mesmo considerado um método (BARTON, 2007, p. 29-68), geralmente as abordagens diacrônicas estão enquadradas dentro de um leque abrangente conhecido como método histórico-crítico. Porém, é importante reconhecer que o método histórico-crítico não surge do vácuo, mas como parte do desenvolvimento natural de diversos movimentos (p. ex., Renascença, Reforma Protestante, Iluminismo e a Revolução Científica) que proporcionaram uma certa autonomia filosófica em relação ao domínio da autoridade da religião centralizada manifesta através da Igreja Católica Romana. A combinação desses fatores importantes desempenhou papel crucial para abrir as portas para os métodos histórico-críticos que até recentemente dominou a pesquisa bíblica marcada por abordagens diacrônicas (KRENTZ, 1975, p. 6-32; LAW, 2012, p. 25-80).

\section{Crítica das Fontes}

Antes de tratar da aplicação da crítica das fontes em Jeremias é importante tecer alguns esclarecimentos com objetivo de evitar confusão. O termo

\footnotetext{
Posturas mais conservadora tendem a apresentar grande resistência a crítica bíblica sob o argumento de que as sagradas Escrituras são tratado de forma tendenciosa por estudiosos que tem o objetivo de trazer descrédito (cf., LINNEMANN, 1986), porém isso não reflete o interesse da maior parte dos interesses acadêmicos que apenas procurar oferecer o mesmo tratamento para o texto bíblico dado a outros textos clássicos da antiguidade. Para maiores detalhes recomendo a leitura de BARR, 1977, p. 40-89, 120-59 e SPARKS, 2008, p. 57-132.
} 
Quellenkritike é usado de forma frequente como sinônimo de Literarkritik $^{6}$ na literatura alemã, ${ }^{7}$ porém como Barton alerta, o termo crítica literária não deve ser confundido com a percepção da literatura francesa i.e. explication de texte, ${ }^{8}$ onde o leitor tenta abordar o texto como uma obra de arte literária procurando desvendar o texto de acordo com a sua coerência interna em conjunto com as técnicas e estilos de composição utilizados pelo autor (BARTON, 1996, p. 20-22). Outro ponto importante é o fato de que ocasionalmente o termo alta crítica é usado para se referir a crítica das fontes (principalmente na literatura inglesa mais antiga) em contraste a crítica textual também tratada como baixa critica (BARTON, 1996, p. 21).

Mudanças paradigmáticas concernente aos estudos veterotestamentários ocorreram inicialmente com a implementação do método histórico-crítico nos estudos do Pentateuco e nos Profetas Anteriores. Graf e Wellhausen empregaram a crítica das fontes propondo que o Pentateuco é composto por quatro fontes: Jehovist, Elohist, Deuteronomiums e Priesterkodex (WELLHAUSEN, 1899, p. 1-14). Dessa forma, é possível reconhecer que estudos em Jeremias não ocorreram de forma isolada deste contexto uma vez que eles devem uma dívida enorme ao legado dos seus percussores modernos. Nesse ponto Duhm é amplamente reconhecido dentro da erudição bíblica como aquele que inaugurou os estudos críticos modernos aplicados a Jeremias sob o modus operandi de Wellhausen propondo que o livro é composto por três fontes: "die prophetischen Gedichte Jeremias", "das Buch des Baruch" e "die Ergänzungen zu den Schriften Jeremias und Baruch") sugerindo que o livro "ist also langsam gewachsen, fast wie unbeaufsichtiger Wald wächst und sich ausbreit" (DUHM, 1901, p. xi-xx). De acordo com o seu critério baseado no ritmo 3/2, Duhm considerou que apenas 280 palavras (a maior parte em forma de poesia lírica) poderiam ser atribuídas ao Jeremias histórico sendo que outras 220 (com o gênero semelhante a crônicas, porém fora da ordem original) foram creditadas a Baruque e os demais 850 versos (que se assemelham a teologia retributiva presente na Torah tiveram sua historicidade questionadas sendo considerados de teor teológico) foram considerados como acréscimos (DUHM, 1901, p. xii-xvi).

\section{Crítica das Formas}

Enquanto Wellhausen foi claramente a principal figura na primeira onda da abordagem histórico-crítica por meio da crítica das fontes, não

Crítica das fontes.

Crítica literária.

BERLEJUNG, 2012, p. 34-35. Habel (1971), por exemplo, utiliza o termo Literary Criticism.

Explicação do texto. 
seria exagero dizer que Gunkel passou a exercer o papel de protagonista na segunda onda sendo considerado o pai da Formkritik/Gattungskritik além de ter o seu nome fortemente associado a escola da história das religiōes ${ }^{9}$. A crítica da forma pode ser entendido como um estágio além das fontes e busca avaliar os estágios pré-literários do material bíblico usando dois conceitos chaves (Gattung e Sitz im Leben ${ }^{19}$ ) nesse método. O primeiro deles, Gattung diz respeito a gêneros literários aplicados a pequenas pericopes com o foco em formas de comunicação oral. O segundo, Sitz im Leben, trata do contexto vital em que esses gêneros literários ocorrem (BARTON, 1996, p. 30-34). Se Duhm foi visivelmente influenciado por Wellhausen, é nítido o impacto que Gunkel exerceu sobre Mowinckel devido as homenagens dedicadas ao seu mentor (MOWINCKEL, 1914, p. 14, 66-67). Embora Mowinckel nunca tenha abandonado a crítica das fontes, ele claramente beneficiou-se das contribuições oferecidas pela crítica das formas e avançou os estudos em Jeremias. Isso explica alguns pontos em comum com seu percussor Duhm bem como divergências. Isso fica claro quando ele critica seus contemporâneos por meramente reproduzir na literatura profética os mesmos métodos aplicados ao Pentateuco ao argumentar que "die Prophetenbücher sind Sammlerwerke, wie es die Evangelien sind” acrescentando que os oráculos proféticos:

betrifft, so ist dies weder eine wohlgegliederte, logisch geordnete Rede, noch ein Aufsatz; es ist eine ekstatische Vision, eine enthusiastisch fließende Rede; es bewegt sich nicht in Begriffen, sondern in anschaulichen Bildern, in halb mystischen Andeutungen, in krampfhalf zuckenden, lose aneinander gereihten Worten. Das echte Orakel hat keine Disposition, keine vorwärts schreitende Gedankenfolge; die Bilder, die charakteristischen Züge des geschilderten zukünftigen Zustandes sind kaleidoskopisch durcheinander gewürfelt (MOWINCKEL, 1914, p. 3-4).

Na percepção de Mowinckel, "das eigentliche Buch Jeremia umfaßt nur die Kapitel 1-45” entendendo que "die Kapitel 46-52 sind ein späterer Anhang, etwa wie Jes. 40-66" com a seguinte conclusão: "Wir wenden uns num zu dem eigentlichen Buch Jeremia, dem Werk des RJ. Wir wollen hier den Beweis dafür antreten, daß das Buch Jer. 1-45 aus vier verschiedenen schriftlichen Quellen zusammengearbeitet ist" (MOWINCKEL, 1914, p. 1417). A influência de Gunkel se faz presente na forma que Mowinckel trata das duas fontes, pois diferente de Duhm, ele entende que estilo por si mesmo não é evidência suficiente para atribuir a uma fonte específica argumentando que:

\footnotetext{
9 Enquanto a sua obra em Gênesis (GUNKEL, 1922) deu atenção especial as lendas nos bastidores das fontes introduzidas por Wellhausen, seu trabalho no saltério (GUNKEL, 1933) focou em diferentes tipos de materiais dos quais pertencem à mesma família.

10 Crítica das formas e contexto vital.
} 
daß eine Stiluntersuchung an sich keine Anhaltspunkte für eine quellenscheidende Kritik geben kann. Da nun aber die prophetische, wie überhaupt die hebräische, Literatur immerhin mit einer recht beschränkten Zahl Stilarten arbeitet, und da diese so fest und typisch sind, so liegt es auf der Hand, daß jede Änderung in der Art der Wiedergabe der Orakel und in der Auffassung des eigentlichen Prophetischen leicht erkennbar sein wird (MOWINCKEL, 1914, p. 18).

As diferenças entre Mowinckel e o seu antecessor ficam mais evidentes com a sua crítica ao dogmatismo de Duhm. Pois ele impõe que "Jir. nur das Fünfermetrum gebrauchen darf" (MOWINCKEL, 1914, p. 20-22), e revela um critério mais flexível em relação a sua fonte $\mathrm{A}$ da qual representa $\mathrm{O}$ trabalho de um colecionador que fielmente preservou as palavras do profeta que se encontram entre Jr 1-25. Nas palavras de Mowinckel:

abgesehen von einigen späteren Zusätzen und zufälligen und unvermeidlichen Textverderbungen macht die Sammlung A den Eindruck, authentische, redaktionell wenig bearbeitete Überlieferung zu sein; sie hat fast durchgängig die echt prophetische, metrisch-rhythmische Form; die meisten ihrer Orakel geben sich durch Form und Inhalt als Werke eines und desselben Mannes zu erkennen; dieser Mann zeigt sich als eine besonders ausgeprägte, von den meisten anderen Propheten deutlich verschiedene Individualität. Also: wenn je, dann haben wir in A ipsissima verba Jeremiae. Wenn wir nun in unserem Buche Jer. eine äußerlich abgetrennte, für sich stehende Gruppe durch innere und stilistische Merkmale gekennzeichneter Orakel, die alle Zeichen einer wortgetreuen Überlieferung an der Stirn tragen, neben einer Gruppe Erzählungen, in denen der Wortlaut der gesprochenen Worte eine mehr nebensächliche Rolle spielt (s. unten), finden, so haben wir fraglos das Recht, anzunehmen, daß diese Gruppen auch selbständige Sammlungen und somit selbständige Quellen darstellen. Unsere Gruppe A ist nicht lediglich eine von uns willkürlich ausgeschiedene Gruppe Orakel derselben literarischen Gattung — sie umfaßt vielmehr viele Gattungen — , sondern sie ist literaturgeschichtlich ein Werk für sich, das später mit anderen Werken zusammengearbeitet worden ist. Die Sammlung A ist, literarisch betrachtet, nichts als eine Sammlung. Von Ordnung, Einteilung, geschichtlichen und biographischen Notizen, kurz von einer selbständigen Verfasserwirksamkeit des Sammlers finden sich nur geringe Spuren. Zweck der Quelle A ist einfach: eine möglichst vollständige Sammlung jirmejanischer Orakel zu geben. Weiter nichts. Person, Geschichte, Zeit, Lebensumstände des Propheten aufzuklären, seine Umwelt zu schildern oder anzudeuten, daran denkt der Sammler gar nicht; er will lediglich die Weissagungen mitteilen (MOWINCKEL, 1914, p. 20-22).

\section{Em relação à fonte $\mathrm{B}$, Mowinckel a descreve como:}

Der Stil dieser Stücke ist die von der Sagenerzählung beeinflußte geschichtliche Erzählung, die wir z. B. aus den Büchern Samuelis kennen. Eine geschichtliche Erzählung, nicht wie sie die Annalisten und die offiziellen Historiographen des Hofes und des 
Tempels schreiben, sondern wie sie das Volk, oder die berufsmäßigen Erzähler erzählen. Keine pragmatische Geschichtsschreibung wie sie Herodot als der Erste geschaffen und Thucydides sie ausgebildet hat. Eine solche gibt es im ganzen A. T. nicht, wenn es auch in späterer Zeit häufig behauptet wird; auch die künstlerische und wissenschaftliche Stufe der isländischen Sagas hat die alttestamentliche 'Geschichtsschreibung' bei weitem nicht erreicht. Was diese von der griechischen oder nordischen unterscheidet, ist vor allem, daß sie nur eine Geschichtenerzählung, letztere aber Geschichtserzählung ist (MOWINCKEL, 1914, p. 24).

Embora Mowinckel concorde com Duhm que A e B em grande parte se diferem em termos de gêneros, Mowinckel argumenta que o fator que as definem como fontes distintas não é o gênero, mas sim o conteúdo uma vez que é possível encontrar casos em que A aparece em forma rítmica ( $p$. ex., Jr 1:4-10) além de casos em que B não ocorre em forma de prosa narrativa (p. ex., Jr 43:8-13). Estas distinções entre as fontes levam Mowinckel a diferencia-las as como:

A will eine möglichst vollständige Sammlung der Weissagungen geben, B möglichst viele Notizen und Mitteilungen über Jir. selbst. A erstrebt nur Vollständigkeit in der Überlieferung der Worte; B dagegen will zeigen, wie Gottes Wort und der Heldenmut und die Treue des Propheten sich betätigen und ihr Recht behalten; A gibt nur eine lose Aneinanderreihung; B erstrebt, wenn auch nicht immer mit Glück, ein zusammenhängende und chronologisch geordnete Erzählung, A ist das Werk eines Redaktors, B das eines Verfassers (MOWINCKEL, 1914, p. 20, 27-28).

No entanto é importante ressaltar que Mowinckel mudou a sua posição original em relação a fonte $\mathrm{B}$. Inicialmente ele defendia que essa coleção de narrativas escritas anonimamente era originalmente oral e foi provavelmente escrita em algum momento após a invasão de Jerusalém e a fuga para o Egito (MOWINCKEL, 1914, p. 28-31). Porém, mais tarde, Mowinckel passou a aceitar a possiblidade de que Baruque poderia ser o melhor candidato para autoria dessa coleção (MOWINCKEL, 1946, p. 61). Semelhantemente Mowinckel inicialmente argumentou que a fonte $C$ poderia ser encontrada espalhada entre as fontes A e B sugerindo que:

Der Beweis, daß hier eine selbständige Quelle vorliegt, läßt sich womöglich noch sicherer als bei A und B führen. Wenn wir nachweisen können, daß die Stücke dieser Gruppe gemeinsame Eigentümlichkeiten aufweisen, sowohl stilistisch als inhaltlich, daß sie eine besondere Eigenart in der Auffassung und Wiedergabe der prophetischen Orakel haben; wenn ferner diese Gruppe Parallelen zu A oder B oder zu beiden aufweist, dann ist der Beweis geführt (MOWINCKEL, 1914, p. 31). 
Um dos seus critérios para a fonte $C$ baseava-se na presença de cabeçalhos contendo dois aspectos: "den göttlichen Ursprung" e "das Datum des betreffenden Wortes, bezw. der Rede angeben, so sind jene die möglichst kurzen Inhaltsangaben" (MOWINCKEL, 1914, p. 32-33) acrescentando a seguinte sugestão em relação a.C.:

Die nicht vielen übrigen Überschriften des Buches Jir. sind entweder ganz kurze Inhaltsangaben, oder Sammelüberschriften, oder unechte Glossen. Schon dadurch werden die ausgeschiedenen Stücke zu einer äußeren Einheit zusammengebunden und von A und B unterschieden; in A treten nur ganz vereinzelte Inhaltsangaben auf, und B hat als eine gewissermaßen fortlaufende Erzählung keine Überschriften (MOWINCKEL, 1914, p. 32-33).

Do ponto de vista linguístico, os discursos presentes em C são monótonos assemelhando-se a “'deuteronomischen' Sprache der redaktionellen Partien von Dtn” (MOWINCKEL, 1914, p. 33-34). Apesar de eventuais semelhanças entre as fontes $\mathrm{A}, \mathrm{B}$ e C, é possível constatar diferenças significativas, pois embora B e C ocorrem em forma de prosa, A e B demonstram interesses respectivos em "die biographischen und geschichtlichen Daten” enquanto C foca em "die Reden des Jir" (MOWINCKEL, 1914, p. 34-35). Mesmo que exista pontos de intersecção entre A e C em termos de conteúdo (p. ex., discurso de reprovação a idolatria), a fonte $\mathrm{A}$ geralmente expõe o tema em forma de oráculos enquanto $\mathrm{C}$ o faz à semelhança do Deuteronômio (MOWINCKEL, 1914, p. 34-45). No entanto, Mowinckel posteriormente desenvolveu sua opinião sobre a relação entre as tradições oral e tradição escrita argumentando que "in the transmission of the sayings of Jeremiah and of the tradition about him, therefore, [...] oral and written tradition have from the beginning gone hand in hand together" (MOWINCKEL, 1946, p. 34-35), com isso ele contribuiu de maneira decisiva para a sua mudança de opinião em relação a sua fonte $\mathrm{C}$ da seguinte maneira:

These prose speeches are neither planned literary, "editorial" adaptations of an existing book, as held by Duhm, nor as a separate literary "source", as previously maintained by the present author, but they represent exactly a circle of tradition of their own, within which certain of the sayings by Jeremiah have been transmitted and transformed according to the ideas and the style which prevailed in the circle, exactly the deuteronomistic ideas and forms of style and interests (MOWINCEKL, 1946, p. 62).

A última fonte da proposta de Mowinckel (D) está localizada entre Jr 30-31. Ele acredita que ela deve ter sido inserida de forma tardia em $\mathrm{R}^{\mathrm{A}-\mathrm{B}-\mathrm{C}}$ devido o seguinte argumento: "selbständige, in sich abgeschlossene 
Sammlung bilden, geht daraus hervor, daß sie inhaltlich einen einheitlichen Gedanken zum Ausdruck bringen und ein besonderes, Thema erschöpfen wollen" (MOWINCKEL, 1914, p. 45-46, 64-65). Mowinckel acrescenta: "sind die aufzuzeichnenden Worte als Heilsweissagungen gekennzeichnet" das quais "aufgezeichnet werden als ein Zeugnis für die Zukunft, da Jhwh das Heil bringen wird" (MOWINCKEL, 1914, p. 45-46, 64-65). Diferente da fonte A, Mowinckel entende que "Diese Sammlung D hat gar nicht den Anspruch jirmejanisch zu sein erhoben; sie ist ursprünglich anonym gewesen, trägt keinen Verfassernamen an der Stirn und gibt somit über ihren Verfasser keine Andeutungen" (MOWINCKEL, 1914, p. 45-46, 64-65). Nesse aspecto Mowinckel continua próximo da percepção de Duhm ao dizer que:

Daß der Verfasser von 30,3. 31,26 kein wirklicher Prophet ist und die von ihm mitgeteilten Orakel nicht selbst verfaßt hat, sondern lediglich ein Redaktor und Bearbeiter älterer Weissagungen ist, der den Mantel des Propheten literarisch angelegt hat, ist völlig klar (MOWINCKEL, 1914, p. 47).

Sua principal justificativa para rejeitar a autenticidade desses textos se baseia no fato de que "die Gleichstellung Ephraim = Israel ist zu deutlich" (MOWINCKEL, 1914, p. 47). Ainda no tocante a fonte D, é necessário fazer uma correção em relação ao meu artigo anterior (YAN, 2016, p. 13-30) onde menciono que Mowinckel mudou sua posição em relação a sua fonte $\mathrm{D}$ sendo que ele apenas revisou a sua posição no tocante as fontes B e C. O que ocorreu é que a sua proposta recebeu maior aceitação em termos da divisão clássica A-B-C sendo que a fonte $\mathrm{D}$ não obteve a mesma aprovação. Esse fato pode ser observado no comentário de Rudolph que mantém a divisão de três fontes ("die Sprüche Jeremias", "die Erzählungüber Jeremia" e "Reden Jeremias in deuteronomischer Bearbeitung") apesar de algumas modificações omitindo D (RUDOLPH, 1947, p. xiii-xv) mesmo após Mowinckel mudar a sua visão fazendo a seguinte afirmação:

Es war die von Duhm [...] angebahnte Entdeckung Mowinckels (zur Komposition des Buches Jeremia, 1914), daß es noch eine Gruppe von Abschnitten im Jeremiabuch gibt, die formal und inhaltlich einander so ähnlich sind, daß sie ebenfalls einmal eine Sonderquelle gebildet haben müssen, die jetzt in dem Buche verarbeitet ist. Wir haben demnach 3 große Gruppen zu unterscheiden (RUDOLPH, 1947, xiii-xv).

A mudança de direção na abordagem de Mowinckel lançou as sementes para o que mais tarde passou a ser reconhecido como redação Deuteronomista de Jeremias apesar de que ele mesmo não tenha desenvolvido uma metodologia que possa ser propriamente enquadrada como crítica da redação. 


\section{Crítica da Redação}

Embora a figura do redator sempre estar presente na crítica bíblica moderna, existem variações significativas na forma como disciplinas diferentes fazem referência ao seu papel que precisam ser esclarecidas. De acordo com Stone, na percepção dos críticos das fontes o termo redator era usado "to account for the ultimate conceptual unity of documents such as the Pentateuch which, to all appearances, had emerged from diverse origins", essa visão mais negativa em relação ao papel do redator entendeu que a sua interpretação mais tardia provavelmente tenha distorcido as fontes (STONE, 1997, p. 79) como é devidamente ilustrado pela seguinte afirmação de Duhm:

Nun kann man zwar sagen, dass die dritte Hand, die nachträglich v. i f. vorsetzte, die ursprüngliche gute Disposition von v. 3 ff. über den Haufen geworfen habe; aber dann würde sie nicht so verfahren haben, wie sonst die Ergänzer im B. Jeremia thun, sondern etwa so, wie es die Redaktoren in der Genesis zu machen pflegen: sie hätte die vorgefundenen Verse nicht angetastet, sondern nur umgestellt (DUHM, 1901, p. 297).

A prioridade histórica na abordagem de Duhm é algo visível no trecho abaixo:

Ein solches Verfahren ist bei historischen Texten leicht zu begreifen, aber im B. Jer wäre es eine Ausnahme; hier greifen die Ergänzer, denen es ja nicht um die Geschichte, sondern um Lehre und Weissagung zu thun ist, in ganz anderer Weise ein; sie sind keine behutsamen Redaktoren, sondern Midraschisten, denen die Erzählungen Baruchs teils als Text, teils nur als Anregung für ihre freien Kompositionen dienen und die sich gar nicht die Mühe geben, den Wortlaut zu bewahren und dann, wenn er nicht recht passt, durch vorsichtige Umstellungen sich brauchbar zu machen (DUHM, 1901, p. 297).

Os pontos de ligação entre Jr 52 e 2 Rs 24:18-25:30 fornecem um exemplo de como redatores tardios podem ter interferido nas fontes levando Duhm a especular que "Der Redaktor, der das that, ist der Meinung gewesen, dass die Zerstörung Jerusalems und des Tempels und die Exilierung des Volkes den wichtigsten Gegenstand bilden, für den man sich beim Lesen des Buches Jeremia interessiere" (DUHM, 1901, p. 377).

Não obstante, de acordo com Stone, a perspectiva dos primeiros críticos das formas considerava o redator como guardião das tradições entendendo que a sua tarefa estava restrita em converter "the material into written text" sem papel criativo. Apesar disso, Stone admite que a análise do processo completo do projeto da Überlieferungsgeschichte ${ }^{11}$ cobria desde o estágio oral até a forma

11 História da tradição. 
final (STONE, 1997, p. 80). Todavia, algumas vezes o papel do redator na disciplina da crítica das formas parece obscuro uma vez que Gunkel menciona que "solche Sammlungen gibt es nun verschiedenartige: entweder werden die ursprünglichen kleinen Einheiten ohne jede Verbindung nacheinander aufgezeichnet" sendo que algumas frases adiante ele menciona que essas coleções aparentam "manchmal in einem komplizierten Prozefs, mehr oder weniger zur Einheit verwoben und mit ihrem Geiste erfüllt sind" (GUNKEL, 1906, p. 1863-1865). Ele diz isso antes de fazer a seguinte afirmação:

Zum Schlufs dann die Tragödie der israelitischen Literatur: der Geist nimmt ab; die Gattungen sind verbraucht; Imitationen häufen sich; an Stelle der originalen Schöpfungen treten die Bearbeitungen; die Sprache stirbt als Volkssprache aus. Aber schon hat die Geschichte der Sammlung der Sammlungen begonnen: der Kanon entsteht (GUNKEL, 1906, col. 1863-1865).

O conflito entre a preservação e a transformação de tradições anteriores pode ser observado no contraste entre a primeira e a segunda obras de Mowinckel, pois embora ele não atribua sua fonte A ao Jeremias histórico, ele a considera como uma coleção que preservou fielmente a ipssissima verba Jeremiae por meio de $\mathrm{R}^{\mathrm{a}}$ (MOWINCKEL, 1914, p. 21-22). Por outro lado, sua reavaliação do material $\mathrm{C}$ parece seguir a linha que as tradições foram transformadas durante o processo de transmissão (MOWINCKEL, 1946, p. 62). Diferentemente Rudolph parte para uma outra direção ao não considerar o "Reden Jeremias in deuteronomischer Bearbeitung" uma distorção das palavras do profeta (RUDOLPH, 1947, p. xiii-xv), pois ele argumenta que:

daß sie andererseits keine freieti Schöpfungen sind, sondern auf echten Aussagen Jeremias fußen, ergibt sich nicht nur daraus, daß mehrmals $\left(\begin{array}{l}7 \\ 28\end{array}\right.$ f. $\left.11_{15} \mathrm{f} .16{ }_{16} \mathrm{f}.\right)$ ursprüngliche Jeremiaworte wörtlich aufgenommen werden, sondern auch daraus, daß in der Hälfte der Fälle $\left(11_{5}\right.$ f. ${ }_{9} 16,1{ }_{1} 11_{3}{ }_{3}{ }_{5} 35$ ff. $)$ Selbstberichte Jeremias zugrunde liegen, die aber nicht der Quelle A zugerechnet werden können, eben weil sie die deuteronomische Bearbeitung aufweisen (RUDOLPH, 1947, p. xiii-xv).

É provável que a migração de uma abordagem mais atomística para uma visão mais orgânica do texto bíblico por parte de Mowinckel tenha sido influenciada pela escola da história da tradição. Apesar de Mowinckel já ter empregado o termo Überlieferungsgeschichte, ele mesmo não chegou a desenvolver uma metodologia que pode ser propriamente considerada como crítica da redação. Porém é justo dizer que se Komposition diagnosticou as limitações da crítica das fontes, certamente Prophecy and Tradition começou a detectar os limites da análise oferecida pela crítica das formas. Segundo Mowinckel, não 
oferece uma síntese devido uma falta da visão integral dos livros proféticos (MOWINCKEL, 1946, p. 42-60).

Nessa altura é necessário observar que de forma curiosa o termo Redationskritik/Redaktionsgeschichte ${ }^{12}$ ocorre com mais frequência nos estudos neotestamentários do que nas pesquisas veterotestamentárias que por sua vez emprega com maior regularidade o termo Überlieferungsgeschichte. Stone acredita que é possível que esse fenômeno tenha ocorrido devido o intercâmbio de idéias entre Rad e seus colegas da área dos estudos neotestamentário (STONE, 1997, p. 80-81). Não obstante Rylaardsdam e Barton entendem que ambas usufruem dos resultados e contribuições apresentados pela crítica das fontes e das formas com o intuito de oferecer uma síntese do processo completo de composição (RYLAARDSDAM, 1972, p. vii; BARTON, 1996, p. 46-47). Apesar do interesse comum por parte das duas disciplinas, talvez a principal diferença seja que a crítica da história da tradição enfatiza o papel da transmissão oral. Embora Nielsen argumenta que o papel da tradição oral não deve ser reduzido ao estágio pré-literário uma vez que as tradições orais e tradições escritas podem coexistir lado a lado (NIELSEN, 1954, p. 12-13). Stone acrescenta que o processo de conversão da tradição oral em documentos escritos "involved much more than mere 'scissors and paste' work, but flowed out of a profound interpretative mindset" incluindo "the impact of the successive reordering undergone by the tradition as the literary documents were compiled and edited" (STONE, 1997, p. 81). De forma parecida Kratz define essa abordagem como "die Berbeitung eines vorgegebenen Texts im Rahmen der schriftlichen Überlieferung und dessen Umgestaltung zu einem neuen Ganzen" (KRATZ, 1997, p. 367) complementando com o seguinte pensamento:

Redaktionskritik bezeichner dementsprechend das Bemühen um die begründete Unterscheidung von Vorlage und Redaktion sowie um ein an den Texten nachvollziehbares Verständnis der dabei herrschenden Bearbeitungs - und Gestaltungsprinzipien, Redaktionsgeschichte den Vorgang der Redaktion als Prozeß der Texttenstehung in ihrer literarischen und sachlichten Dimension (KRATZ, 1997, p. 367).

Nesse sentido, Collins explica que a crítica da redação tenta entender como um corpo de material literário foi organizado, adaptado e transformado em um livro cujo processo é frequentemente entendido como redação/ edição enquanto as figuras responsáveis por essa atividade são chamadas de redatores/editores (COLLINS, 1993, p. 15).

12 Também chamado frequentemente de Literaturgeschicbte cf. SCHMID, 2008. 
A síntese tão almejada por Mowinckel parece ser abordada na nova metodologia apresentada por Rad e Noth. Sendo que Rad iniciou seu procedimento identificando primeiramente os estágios mais primitivos até chegar ao Endstatium. ${ }^{13}$ Enquanto Noth aplicou o processo inverso para posteriormente repetir o mesmo procedimento proposto por Rad (KNIGHT, 2006, p. 77, 130-134). Apesar de reconhecer fontes múltiplas independentes no Hexateuco, Rad entendeu que essas tradições foram unificadas sob um Kredo ${ }^{14}$ no qual o seu Sitz in Leben original acabou sendo incorporado na interpretação Javista da Heilsgeschichte ${ }^{15}$ promovendo o redator de mero compilador a autor com liberdade e criatividade significativa para transformar antigas tradições de acordo com sua agenda teológica (RAD, 1938, p. 37-68). Noth também abandou uma postura fragmentária em relação ao texto bíblico tratando-o como uma unidade literária considerando o colecionador dessas tradições como um autor. Assim, o livro de Deuteronômio não deveria mais ser visto apenas como adendo do Pentateuco, mas sim o ponto de partida que da denteronomistische Geschichtswerk ${ }^{16}$ envolvendo o grande complexo literário cobrindo de Josué a 2 Reis tendo sido completado c. 600 AEC (NOTH, 1963, p. 91).

Rendtorff, no entanto, argumenta que apesar do termo Überlieferungsgeschichte já aparecer nas obras Gunkel, Mowinckel e Rad, Noth merece o crédito de fundador desse método uma vez que ele foi o primeiro a cunhar o termo técnico (RENDTORFF, 1994, p. 93). Mesmo assim é inevitável reconhecer que as contribuições de Rad em relação ao papel do redator ao lado da noção de história Deuteronomista de Noth tiveram impacto profundo no conceito de redação Deuteronomista do livro de Jeremias. Essa influência promoveu uma nova mudança paradigmática em relação a Duhm e Mowinckel que outrora defenderam que Jeremias é fruto de um desenvolvimento sem qualquer planejamento (DUHM, 1901, p. xx; MOWINCKEL, 1914, p. 3). Dessa forma a proposta de uma redação Deuteronomista de Jeremias surgiu inicialmente em dois modelos distintos na América do Norte e na Alemanha. Apesar de que Hyatt que merece o crédito de pioneiro em relação a redação Deuteronomista do livro ${ }^{17}$.

Ao identificar a ameaça do norte (זופז) com os babilônios Hyatt rejeitou sugestões antigas que procuravam identificar o inimigo do norte como al-

\footnotetext{
Estágio ou forma final.

Credo.

História da salvação.

6 Trabalho do Deuteronomista.

17 A proposta de Hyatt se encontra espalhada em diversos artigos, porém é possível encontrar duas versões resumidas da sua tese em HYATT, 1951, p. 71-95; 1956, p. 777-93.
} 
gum dos povos que invadiram do antigo Oriente Próximo ${ }^{18}$. Segundo Hyatt, o profeta Jeremias foi sincronizado ao período do rei Josias. Ele argumenta que Jr 11 "has undoubtedly received expansion at the hands of an editor of the Deuteronomistic school who wished to make it appear that Jeremiah had supported Josiah's reforms" (HYATT, 1940, p. 511-512). Presumivelmente, o centro do modelo da redação Deuteronomista de Hyatt é construída em grande parte em cima da interpretação do Jr 11 ao qual ele argumenta que esse capítulo ao lado de passagens como Jr 3:6; 25:3 e 36:2 são claramente produto de tradições tardias (HYATT, 1941, p. 57-60). A principal barreira para Hyatt aceitar a contemporaneidade entre Jeremias e o rei Josias está baseada no fato de que ambos apresentam teologias opostas na qual o profeta parece empregar o termo Torah em termos éticos baseado no decálogo dentro da linha da religião praticada no deserto que é antagônico a religião ritualística do templo (HYATT, 1941, p. 381-397). Na percepção de Hyatt a religião do profeta reflete uma espiritualidade internalizada onde leis sinaíticas são colocadas em prática promovendo justiça social à semelhança da mensagem de Oséias (HYATT, 1943, p. 95-96). Ao tentar explicar a relação entre os trabalhos de Jeremias, o Deuteronômio original e os Deuteronomistas, Hyatt argumenta que o profeta teria conhecimento da versão original de Deuteronômio e que as versões originais de Jeremias e Deuteronômio apresentam linguagem semelhantes que podem ser explicadas no sentido de que ambos foram compostas cerca do mesmo período entre o sétimo e começo do sexto século AEC (HYATT, 1942, p. 163-165). Posteriormente, os editores Deuteronomistas procuraram apresentar o profeta como simpatizante da reforma utilizando-se das seguintes técnicas editoriais (HYATT, 1942, p. 165-73):

1. Datas em Jeremias com a intenção colocar o início da carreira pública do profeta antes da reforma Deuteronomista.

2. Passagens inseridas para enfatizar que os pecados pré-Deuteronômicos anteriores a fase inicial do ministério profético de Jeremias.

3. A composição de Jr 11:1-8 para provar que Jeremias era apoiador da reforma Deuteronomista.

4. Passagens elaboradas para explicar a causa do exílio como resultado da desobediência a Javé e como consequência da idolatria.

18 HYATT, 1940, p. 499-513. Por ex., a hipótese Cita, que era que relativamente popular no princípio dos estudos modernos em Jeremias na qual identificava o inimigo com as invasões oriundas da região da Cítia baseada nas descrições de Heródoto (cf. Hist. I.103-106). Duhm, por exemplo, refere a ameaça do norte como Skythenlieder (cf. DUHM, 1901, p. xiv) enquanto Torrey propôs que o inimigo se tratava da invasão de Alexandre o Grande (cf. TORREY, 1937, 193-216. Outras propostas anteriores também interpretaram a ameaça do norte como um julgamento escatológico, cf. VOLZ, 1922, p. 58 e WELCH, 1928, p. 97-131. 
5. Passagens elaboradas prevendo a restauração e prosperidade após o exílio.

6. Passagens legalistas inseridas com o intuito de provar que o profeta tinha conhecimento das leis do Deuteronômio. Pelo menos em uma ocasião (Jr 28), Hyatt acredita que o profeta possa ter sido influenciado pelo Deuteronômio.

Consequentemente, fica claro que Hyatt defende que o profeta Jeremias só poderia ter dado início ao seu ministério após a morte do rei Josias dando luz a um debate com Whitley em relação a data do chamado do profeta ${ }^{19}$. Antes de Thiel apresentar a sua versão de redação Deuteronomista de Jeremias, Nicholson já demonstrava uma mudança de enfoque caminhando para uma direção que abandona o foco no profeta e presta atenção a Jeremias como uma obra literária. Mesmo admitindo que Jr 36 possa ter um núcleo histórico ele sugeriu que "the primary purpose of this narrative $[. .$.$] is theological$ and not, as commentators have so often suggested, merely biographical" (NICHOLSON, 1970, p. 2). Por conseguinte, Nicholson argumenta que o relato do escriba Baruque oferece três aspectos sobre o surgimento e desenvolvimento dos escritos proféticos, em especial Jeremias: (1) a transformação dos primeiros oráculos de Jeremias em escrita é algo que ocorreu em um estágio relativamente cedo por meio do próprio profeta. (2) os acréscimos na coleção original após o rolo original ter sido queimado pelo rei Joaquim e a importância do papel de Baruque (NICHOLSON, 1970, p. 2-3). Essas observações preliminaries levam Nicholson a argumentar que Jeremias é o resultado de "a purely or predominantly literary activity" no qual a sua "present form as well as much of the material in it to scribal and literary activity of Baruch and other authors and editors" (NICHOLSON, 1970, p. 3). Semelhante a reconsideração de Mowinckel em relação aos discursos em prosa Nicholson apresenta uma proposta paradoxal em termos da presente forma de Jeremias onde ele argumenta que a forma presente do livro não reflete meramente a transmissão das palavras do profeta, mas para apresentar uma interpretação do seu ministério profético junto a sua pregação:

On the basis of theological concerns and interests which were of vital importance for them in the age in which they lived. Such a tradition would have emerged and evolved not at the hands of individual authors and editors but within the context of an active preaching and teaching ministry which addressed itself to a listening audience, just as

19 Embora Hyatt já tenha argumentado sobre o início do ministério de Jeremias em 1940, ele expande seu ponto em relação a cronologia do início da carreira do profeta em resposta a Whitley que sugeriu que o inicio do ministério profético do profeta tenha se iniciado após a batalha de Carquemish (cf. HYATT, 1966, p. 204-18; WHITLEY, 1964, p. 467-83; 1968, p. 38-49). 
the preaching of Jeremiah himself and the Word of God which he proclaimed were at every stage addressed to an audience. This means that although much of the material in the book can be attributed directly to Jeremiah himself, that is, preserves his ipssissima verba, we must also reckon with the probability that much of it owes its origins and composition directly to the circle of traditionists (NICHOLSON, 1970, 4, 11-16).

Segundo Nicholson, a forma como o círculo dos guardiões da tradição transmitiu a mensagem do profeta segue o mesmo princípio adotado por aqueles que entregaram o conteúdo da história Deuteronomista. Essa reforma está alinhada com o método homilético disponível no Deuteronômio atualizando e contextualizando as palavras proféticas para a presente geração (NICHOLSON, 1970, p. 7-10). Um ponto de ruptura entre Nicholson e os pais da crítica moderna em Jeremias (Duhm e Mowinckel) é o apontamento de que nenhuma das narrativas individuais nos capítulos 26-36 podem ser de fato classificadas como parte constituinte de uma Leidensgeschichte ou mesmo consideradas como uma biografia geral uma vez que em cada um dos casos seu propósito principal não é biográfico tão pouco histórico, mas sim com fins didáticos. Assim é bem provável que elas tenham se desenvolvido de forma parecida com os sermões e discursos. Isso significa que esse material deve ser entendido como estórias edificantes das quais suas origens pertencem ao círculo dos tradicionistas que as elaboraram com o objetivo de lidar com as implicações dos diversos incidentes que sucederam o ministério e ensino do profeta para atender suas próprias preocupações, p. ex., a autoridade da palavra profética, o problema da falsa profecia e a desobediência e as exigências da Lei (NICHOLSON, 1970, p. 2-16).

Mesmo que Thiel tenha reconhecido o mérito da proposta da edição Deuteronomista por parte de Hyatt reconhecendo como "als beste Erklarung der Tatbestände angesehen werden dürfen” (THIEL, 1973, p. 3-31), ele levanta problemas potenciais. Por exemplo "Die sich an diesem Punkt erweisende fehlende Differenzierung zwischen 'deuteronomisch' und 'deuteronomistisch' que ainda continua assumindo "die Entstehung des Dtn. sehr stark an die Josia-Zeit heranzurücken” (THIEL, 1973, p. 3-31) Consequentemente, as conjecturas de Hyatt acabam levantando problemas maiores dos quais Thiel resume da seguinte forma:

Wenn man hingegen das Dtn. mehr als ein halbes Jahrhundert früher im Gebiet des ehemaligen Nordreichs entstanden denkt, wird man eher geneigt sein, zwischen seinem ursprünglichen Anliegen und seiner judäischen Rezeption, aber auch zwischen dieser und ihrer Neuauflage im Exil zu unterscheiden. Es ist wesentlich plausibler, daß die Tätigkeit des dtr. Kreises im Exil an das noch vorhandene Dokument, das Dtn., anknüpfte - freilich in eigener, dtr. Interpretation — als an das zurückliegende Ereignis der 
josianischen Reform. Die Motive der Redaktion können also schwerlich so literarisch und einseitig biographisch auf das Dtn. und die josianische Reform bezogen werden. Hinter diesem Gesichtspunkt tritt für HYATTS nämlich die Exilssituation der Texte stark zurück, damit aber auch der Predigtcharakter der Reden und ihre homiletische Situation (THIEL, 1973, p. 31).

Enquanto o modelo editorial proposto por Hyatt está limitado a passagens específicas de Jeremias, a proposta de Thiel sugere que Jeremias tenha passado por um programa editorial detalhado nos bastidores do livro como um todo. Thiel concentra sua investigação nos capítulos 1-25, onde as palavras do profeta estão contidas, sugerindo o seguinte:

Die erste Aufgabe stellt die Identifikation und Aussonderung des redaktionellen Materials dar. Obwohl hier sehr wichtige — z.T. jedoch von anderen Voraussetzungen her geschriebene - Arbeiten vorliegen, herrscht über die Abgrenzung der betreffenden Texte in der Forschung, die prinzipiell die Evidenz dtr. Texte im Buche Jeremia bejaht, alles andere als Einigkeit. So wird die Hauptarbeit darin bestehen, in dieser Frage zu einem möglichst gesicherten und überzeugenden Ergebnis zu gelangen. Im engsten Zusammenhang damit steht die Herausarbeitung des Redaktionsverfahrens, d.h. der Arten und Möglichkeiten, mit der die Verknüpfung der vorgegebenen Überlieferungen von der Redaktion vollzogen wurde. Dabei wird auch die Frage nach der Verarbeitung des zugrundeliegenden älteren Materials zu klären sein. Gerade durch die Aufdeckung derartiger 'Kristallisationspunkte' kann man hoffen, die Arbeitsweise der Redaktion besonders gut in den Blick zu bekommen. Schließlich dürfte sich besonderes Interesse auf die leitenden Prinzipien dieser Redaktion richten. Derartige Prinzipien, seien es literarische Grundsätze, seien es übergreifende sachliche Gesichtspunkte, stellen ja das für eine Redaktion konstitutive Merkmal dar. Die Aufdeckung dieser Leitgedanken und der hinter ihnen stehenden theologischen Konzeption würde die angesichts der Textverhältnisse evidente These von einer dtr. Redaktion zur Gewißheit erheben. So sehr sich der Nachweis einer dtr. Redaktion auf den Vergleich mit der Literatur dtr. Herkunft berufen muß, so sind doch die Ergebnisse einer solchen redaktionsgeschichtlichen Analyse nicht an Texten außerhalb des untersuchten Buches letztgültig nachprüfbar. Sie müssen sich also schließlich durch Konsequenz der Argumentation, innere Wahrscheinlichkeit and Nachprüfbarkeit an den der Untersuchung unterworfenen, in Wahrheit diese aber dirigierenden Texten selbst legitimieren (THIEL, 1973, p. 44-45).

Thiel conclui que a maior preocupação do editor de Jeremias se resume no seguinte: "Sie interpretiert die Katastrophe von 587 (wie die Nordisraels von 722/21, vgl. II. Kön. 17 fff.) als" (THIEL, 1973, p. 301).

\section{Conclusões}

Abordagens atomísticas representadas por Duhm e Mowinckel serviram para despertar uma consciência de que Jeremias é fruto de múltipla autoria. 
Porém, as conclusões desse tipo de abordagem foram muito além do que realmente pode ser provado cientificamente indicando um pensamento extremamente mecânico. Nesse ponto a distinção entre baixa e alta críticas não se sustentam uma vez que essas diferenças não fazem jus a relação entre $\mathfrak{b}$ - e $\mathfrak{A l}$-Jeremias. Pois a relação entre as versões grega e hebraica sugerem que as questões envolvendo crítica textual e literárias são mais fluídas do que eram antes imaginadas ${ }^{20}$. Nesse sentido as propostas de Migsch, McKane, Tov e Bogaert apresentam fortes evidências textuais para sustentar suas respectivas sugestões do desenvolvimento literário de Jeremias (MIGSCH, 1981, p. 267; MCKANE, 1986, p. Ixxxiii; TOV, 1997, p. 145-167; BOGAERT, 1997, p. 168-173).

Perspectivas mais orgânicas representadas por Hyatt e Thiel procuram apresentar uma visão mais integral do processo de composição de Jeremias. Porém, a unidade da redação Deuteronômica de Jeremias passou fortemente questionada. Wanke e Migsch apresentaram objeções em relação a unidade dos textos biográficos (WANKE, 1971) uma vez que os textos em prosa prontamente classificados como Deuteronomistas caíram na armadilha do fenômeno pan-Deuteronomismo como solução do tipo Deus ex machina. Porém, Coggins e Lohfink chamam a atenção a importância de reservar o uso do termo Deuteronomista para casos específicos com o objetivo de manter clareza (COGGINS, 1999, p. 22-34; LOHFINK, 1999, p. 36-66). O fato de que muitas vezes o uso da linguagem e estilo Deuteronomista não significa automaticamente que determinados textos devem ser classificados como tal levou Stipp a cunhar o termo Deutero-Jeremiânico para fazer referência a textos que têm a aparência Deuteronomista, porém uma teologia distinta (STIPP, 1998, p. 2 e MAIER, 2017, p. 123). Porém, isso não significa que os Deuteronomistas não tiveram uma participação importante no processo de composição do livro, porém a participação deles é apenas mais um capítulo de um extenso e complexo processo. Certamente os Deuteronomistas exerceram grande influência no corpus profético ${ }^{21}$. Carroll, especificamente, concentra a sua abordagem no papel da ideologia Deuteronomista em parte da composição de Jeremias (CARROLL, 1981, p. 26-30; 1986, p. 65-82). Segundo Stulman, essa etapa Deuteronomista deve ter ocorrido antes da segunda edição agora disponível na edição hebraica do livro, pois os traços da edição Deuteronomista já estão presentes na Vorlage da versão grega (STULMANN, 1982, p. 145). Um outro estágio da redação de Jeremias pode ser observado na contribuição de Polhmann que também critica a unidade da

\footnotetext{
20 Recomendo SÆBØ, 1998, p. 36-46; KRATZ, 1997, p. 369; SCHMID, 2008, p. 33.

21 Recomendo a leitura dos artigos em JACOBS, 2013.
} 
redação Deuteronomista proposta por Thiel (POHLMANN, 1978, p. 16-17). A principal objeção de Polhmann baseia-se no fato de que parte dos textos que com aparência Deuteronomista demonstram inclinação a favor dos exilados na Babilônia (POHLMANN, 1978, p. 183-190). Aqui é necessário fazer uma distinção entre diferentes estágios da redação fazendo uso do termo Fortschreibung ${ }^{22}$ cunhado por Zimmerli nos seu comentário em Ezequiel onde ele faz uso do termo a um estágio mais recente da redação (ZIMMERLI, 1969, p. 109-110; WILLIAMSON, 2013, p. 26-29). No entanto, se admitirmos que a presença de Fortschreibungen em Jeremias aponta para indícios de um estágio editorial relativamente novo em comparação aos demais livros proféticos, é bem possível que Jeremias tenha sido editado a partir deste estágio mais novo vinculado aos demais livros proféticos. Isso sustentaria uma possível dependência literária com Ezequiel, como o Vieweger sugere (VIEWEGER, 1988, p. 15-34). O fato de que (b-Jeremias seguir a mesma estrutura organizacional que os demais profetas maiores (Isaías e Ezequiel) é um dos principais motivos que levaram alguns estudiosos a essa conclusão. Essa distinção entre redação e Fortschreibungen ameniza diferenças drásticas entre as abordagens sugeridas por McKane e aquelas defendidas por Tov e Bogaert. Enquanto McKane se reaproxima de Duhm e Mowinckel sugerindo que Jeremias se desenvolveu sem qualquer planejamento Tov e Bogaert propõe que $\mathbb{6}$ - e $\mathfrak{A l}$-Jeremias refletem duas edições do livro (MCKANE, 1986, p. xlix-lxxxi; TOV, 1997, p. 145-167; BOGAERT, 1997, 168-173).

Jeremias passou por um longo complexo processo de composição no qual demonstra a necessidade de diferenciar a pessoa do profeta em relação ao livro que carrega o seu nome. Embora ainda exista uma produção contínua focada em Jeremias sob uma perspectiva biográfica ou até mesmo engajada na busca da ipsissima verba Jeremiae (LUNDBOM, 2012; STIPP, 2009, p. 148-186), a tendência está voltada para a leitura de Jeremias e os demais escritos proféticos no período Persa ${ }^{23}$.

\section{Referências bibliográficas}

BARR, James. Fundamentalism. London: SCM Press, 1977.

BARTON, John. Reading the Old Testament: method in biblical study. 2. ed. London: Darton, Longman and Todd, 1996.

BARTON, John. The nature of biblical criticism. Louisville, KT; London: Westminster John Knox Press, 2007.

BERLEJUNG, Angelika. Methods. Traduzido por Thomas Riplinger. In: GERTZ, Jan

\footnotetext{
22 Atualização.

23 Recomendo a leitura dos artigos em EDELMAN, 2009 e FISCHER, 2005, p. 120-22.
} 
Christian et al. T\&T Clark handbook of Old Testament. an introduction to the literature, religion and history of the Old Testament. London: T\&T Clark, 2012, p. 19-54.

BOGAERT, P.-M. De Baruch à Jérémie. Les deux rédactions conservées du livre de Jérémie. In: BOGAERT, P.-M. (Ed.). Le livre de Jérémie. Bibliotheca Ephemeridum Theologicarum Lovaniensium LIV. Leuven: Leuven University Press, 1997, p. 168-73.

CARROLL, Robert P. From chaos to covenant: use of prophecy in the book of Jeremiah. London: SCM Press, 1981.

CARROLL, Robert P. Jeremiah. London: SCM Press, 1986, p. 65-82.

COGGINS, Richard. What does "Deuteronomistic" mean? In: SCHEARING, Linda S.; MCKENZIE, Steve L. (Eds.). Those elusive Deuteronomists: the phenomenon of pan-Deuteronomism. Journal for the study of the Old Testament - Supplement series 268, Sheffield, p. 22-34, 1999.

COLLINS, Terence. The mantle of Elijab: the redaction criticism of the prophetical books. The biblical seminar 20. Sheffield: Sheffield University Press, 1993.

DAVIES, Eryl W. Biblical criticism: a guide for the perplexed. London: Bloomsbury, 2013.

DUHM, Bernhard. Das Buch Jeremia. Kurzen Hand-Commentar zum Alten Testament. Tübingen; Leipzig: J.C.B. Mohr, 1901.

EDELMAN, Diana V.; BEN ZVI, Ehud (Eds.). The production of prophecy: Constructing prophecy and prophets in Yehud. London; Oakville, CT: Equinox, 2009.

FISCHER, Georg. Jeremia: 1-25. Vol. 1. Herders Theologischer Kommentar zum Alten Testament. Freiburg im Breisgau: Herder, 2005.

GUNKEL, Hermann. Die Grundprobleme der israelitischen Literaturgeschichte. In: Deutsche Literaturzeitung für Kritik der internationalen Wissenschaft 27, 1906, p. 1863-1865.

GUNKEL, Hermann. Genesis. Göttingen Handkommentar zum Alten Testament. Göttingen: Vandenhoeck \& Ruprecht, 1922.

GUNKEL, Hermann; BEGRICH, Jehoachin. Einleitung in die Psalmen: Die Gattungen der religionen Lyrik Israels. Göttingen: Vandenhoeck \& Ruprecht, 1933.

HABEL, Norman. Literary criticism of the Old Testament. Old Testament series guides to biblical scholarship. Philadelphia, PA: Fortress Press, 1971.

HERSHLER, Moshe; HARAV, Yad (Eds.). The Babylonian Talmud - Tractate Kethubot with variant readings from manuscripts, "Genizah fragments, with quotations from early Rabbinic literature. Jerusalem: Rabbi Herzog World Academy, 1972.

HYATT, J. Philip. The peril from the North in Jeremiah. Journal of Biblical Literature, Atlanta, vol. 59, p. 499-513, 1940.

HYATT, J. Philip. The original text of Jeremiah 11:15-16. Journal of Biblical Literature, Atlanta, vol. 60, n. 1, p. 57-60, 1941.

HYATT, J. Philip. The Torah in the book of Jeremiah. Journal of Biblical Literature, Atlanta, vol. 60, n. 1, p. 381-396, 1941.

HYATT, J. Philip. Jeremiah and Deuteronomy. Journal of Near East Studies, Chicago, vol. 1, n. 2, p. 156-173, 1942.

HYATT, J. Philip. The message of the seventh century prophet for today. Journal of Bible and Religion, Oxford, vol. 11, n. 2, p. 93-97, 1943. 
HYATT, J. Philip. The Deuteronomic edition of Jeremiah. In: BEATTY, Richmond C; HYATT, J. Philip; SPEARS, Monroe K. Vanderbilt Studies in the Humanities. Nashville, TN: Vanderbilt University Press, 1951, p. 71-95.

HYATT, J. Philip. The Book of Jeremiah: Introduction and exegesis. In: TERRIEN, Samuel. The interpreters' Bible commentary. Vol. V. Nashville, TN: Abingdon Press, 1956, p. 777-793. HYATT, J. Philip. Beginning of Jeremiah's prophecy. Zeitschrift für die alttestamentliche Wissenschaft, Berlin, vol. 78, p. 204-218, 1966.

JACOBS, Mignon R.; PERSON JR., Raymond F. (Eds.). Israelite prophecy and the Deuteronomistic History: Portrait, reality, and the formation of a history. Society of biblical literature 14. Ancient Israel and its literature. Atlanta, GA: SBL Press, 2013.

KNIGHT, Douglas A. Rediscovering the traditions of Israel. 3. ed. Society of Biblical Literature 16. Atlanta, GA: SBL Press, 2006.

KRATZ, Reinhard G. Redaktionsgeschichte/Redationskritik. In: BALZ, Horst et al. (Eds.). Theologische Realensyklopäedie. Vol. 28. Berlin; New York: Walter de Gruyter, 1997, p. 367-78. KRENTZ, Edgar. The historical-critical method. Eugene, OR: Wipf and Stock Publishers, 1975.

LAW, David R. The historical-critical method: a guide for the perplexed. London: T\&T Clark International, 2012.

LINNEMANN, Eta. Wissenschaft oder Meinung?: Anfragen und Alternativen. Neuhausen: F. Haenssler, 1986.

LOHFINK, Norbert F. Was there a Deuteronomistic movement?' In: SCHEARING, Linda S.; MCKENZIE, Steve L. (Eds.). Those elusive Deuteronomists: The phenomenon of pan-Deuteronomism. Journal for the Study of the Old Testament - Supplement series 268, Sheffield, p. 36-66, 1999.

MAIER, Christl M. The nature of Deutero-Jeremianic texts. In: NAJMAN, Hindy; SCHMID, Konrad (Eds.). Jeremiah's scriptures: production, reception, interaction, and transformation. Supplements to the Journal for the Study of Judaism 173, Leiden, p. 103-123, 2017. MCKANE, William. Jeremiah. Vol. 1. International critical commentary. Edinburgh: T\&T Clark, 1986.

MIGSCH, Herbert. Gottes Wort über das Ende Jerusalems: Eine literar-, stil- und gattungskritische Untersuchung des Berichtes Jeremia 34,1-7; 32,2-5; 37,3-38,28. Österreichische biblische Studien 2. Klosterneuburg: Österreiches Katholisches Bibelwerk, 1981.

MOWINCKEL, Sigmund. Zur Komposition des Buches Jeremia. Kristiania: J. Dybwad, 1914. MOWINCKEL, Sigmund. Prophecy and tradition: The prophetic books in light of the study of the growth and history of the tradition. Oslo: Jacob Dywad, 1946.

NICHOLSON, E. W. Preaching to the exile: A study of the prose tradition in the book of Jeremiah. Oxford: Basil Blackwell, 1970.

NIELSEN, Eduard. Oral tradition. Studies in biblical theology 11. London: SCM Press, 1954.

NOTH, Martin. Überlieferungsgeschichtliche Studien. Darmstadt: Wissenschaftliche Buchgesellscgaft, 1963.

POHLMANN, Karl-Friedrich. Studien zum Jeremiabuch: Ein Beitrag zur Frage nach der Ents- 
tehung des Jeremiabuches. Forschungen zur Religion und Literatur des Alten und Neun Testaments 118. Göttingen: Vandenhoeck \& Ruprecht, 1978.

RAD, Gerhard von. Das formgeschichtliche Problem des Hexateuch. In: Beiträge zur Wissenschaft von Alten und Neuen Testament 4, 1938, p. 37-68.

REITER, Siefried (Ed.). Sancti Eusebii Hieronymi in Hieremiam Prophetam Libri Sex. Leipzig: Freytag, 1913.

RENDTORFF, Rolf. Martin Noth and Tradition Criticism. In: MCKENZIE, Steven; GRAHAM, M. Patrick (Eds.). History of Israel's traditions: The heritage of Martin Noth. Journal for the study of the Old Testament - Supplement series 182. Sheffield, p. 91-100, 1994.

ROGERSON, John. An outline of the history of Old Testament study. In: ROGERSON, John (Ed.). Beginning Old Testament Study. 2. ed. London: SPCK, 1998, p. 6-24.

RUDOLPH, Wilhelm. Jeremia. Handbuch zum Alten Testament 12. Tübingen: J. C. M. Mohr Paul Siebeck, 1947.

RYLAARDSDAM, J. Foreword. In: RAST, Walter E. (Ed.). Tradition history and the Old Testament. Old Testament series guides to biblical scholarship. Philadelphia, PA: Fortress Press, 1972, p. vii.

SÆBØ, Magne. From pluriformity to uniformity: the emergence of the Massoretic text. In: SÆBØ, Magne (Ed.). On the way to canon: Creative tradition history in the Old Testament. Journal for the Study of the Old Testament - Supplement series 191, Sheffield, p. 36-46, 1998.

SCHMID, Konrad. Literaturgeschichte des Alten Testaments: Eine Einführung. Darmstadt: WBG, 2008.

SPARKS, Kenton L. God's word in human words: an evangelical appropriation of critical biblical scholarship. Grand Rapids, MI: Baker Academic, 2008, p. 57-132.

STIPP, Hermann-Josef. Deutero-Jeremianische Konkordan₹. Arbeiten zu Text und Sprache im Alten Testament 63. St. Ottilien: EOS-Verl., 1998.

STIPP, Hermann-Josef. Sprachliche Kennzeichen jeremianischer Autorschaft. In: BARSTAD, Hans M.; KRATZ, Reinhard G. (Eds.). Prophecy in the book of Jeremiah. Beibefte zur Zeitschrift für die alttestamentliche Wissenschaft, Berlin, vol. 388, p. 148-186, 2009.

STONE, Lawson G. Redaction criticism, wither, and why? Or, going beyond source and form criticism without leaving them behind. In: CARPENTER, Eugene E. (Ed.). A biblical itinerary: In search of method, form and content. Essays in honor of George W. Coats. Journal for the study of the Old Testament - Supplement series 240. Sheffield, p. 77-89, 1997. STULMANN, Louis. The prose sermons of the book of Jeremiah: A redescription of the correspondences with the Deuteronomistic literature in the light of recent text-critical research. Society of Biblical Study Dissertation - Series 83. Atlanta, GA: Scholars Press, 1982.

THIEL, Winfried. Die Deuteronomistische Redaktion von Jeremia 1-25. Wissenschaftliche Monographien zum Alten und Neuen Testament 41. Berlin: Neukirchener, 1973.

TORREY, Charles C. The background of Jeremiah 1-10. Journal of biblical literature, Atlanta, vol. 56, p. 193-216, 1937.

TOV, Emanuel. Some aspects of the textual and literary history of the book of Jeremiah. In: BOGAERT, P.-M. (Ed.). Le livre de Jérémie. Bibliotheca Ephemeridum Theologicarum Lovaniensium LIV. Leuven: Leuven University Press, 1997, p. 145-67. 
VIEWEGER, D. Die Arbeit des jeremianischen Schülerkreises am Jeremiabuch und deren Rezeption in der literarischen Überlieferung der Prophetenschrift Ezekiels. BZ, vol. 32, p. 15-34, 1988.

VOLZ, Paul. Der Prophet Jeremia: übersetzt und erklärt. Kommentar zum Alten Testament. Leipzig; Erlangen: A. Deichert'sche Verlagsbuchhandlung, 1922.

WANKE, Gunther. Untersuchungen zur sogennanten Baruchschrift. Beibefte zur Zeitschrift für die alttestamentliche Wissenschaft, Berlin, vol. 122, p. 1-5, 144, 1971.

WELCH, Adam C. Jeremiah, his time and his works. Oxford: Oxford University Press, 1928.

WELLHAUSEN, Julius. Prolegomena zur Geschchite Israels. 5. ed. Berlin: Druck and Verlarg von Georg Reimer, 1899.

WHITLEY, C.F. Date of Jeremiah's call. Vetus Testamentum, Leiden, vol. 14, p. 467-483, 1964.

WHITLEY, C.F. Carchemish and Jeremiah. Zeitschrift für die alttestamentliche Wissenschaft, Berlin, vol. 80, p. 38-49, 1968.

WILLIAMSON, H.G.M. Redaction vriticism. In: DELL, Katharine J.; JOYCE, Paul M.

Biblical interpretation and method. Essays in honour of John Barton. Oxford: Oxford University Press, 2013, p. 26-36.

YAN, Anderson. Synchronic and diachronic readings on Jeremiah scroll. Vox Scripturae, São Bento do Sul, SC, vol. 24, n. 1, p. 13-30, 2016.

ZIMMERLI, Walther. Ezechiel 1. Biblischer Kommentar Alten Testament XIII/1. Neukirchen-Vluyn: Neukirchener Verlag des Erziehungsvereins GmbH, 1969.

Patrologiae cursus completus: Patrologiae Gracae Tomus XI. Origenes. Petit-Montrouge: Jacques-Paul Migne. 1857.

Submetido em: 30-4-2019

Aceito em: 11-6-2019 